\title{
Catalyzing plant science research with RNA-seq
}

\author{
Laetitia B. B. Martin ${ }^{1}$, Zhangjun Fei ${ }^{2,3}$, James J. Giovannoni ${ }^{2,3}$ and Jocelyn K. C. Rose ${ }^{1}$ * \\ ${ }^{1}$ Department of Plant Biology, Cornell University, Ithaca, NY, USA \\ ${ }^{2}$ Boyce Thompson Institute for Plant Research, Ithaca, NY, USA \\ ${ }^{3}$ Robert W. Holly Center for Agriculture and Health, United States Department of Agriculture-Agricultural Research Service, Ithaca, NY, USA
}

\section{Edited by:}

Alisdair Fernie, Max Planck Institut for Plant Physiology, Germany

\section{Reviewed by:}

Alisdair Fernie, Max Planck Institut for Plant Physiology, Germany

Bjoern Usadel,

Rheinisch-Westfaelische Technische Hochschule Aachen University,

Germany

\section{${ }^{*}$ Correspondence:}

Jocelyn K. C. Rose, Department of Plant Biology, Cornell University,

412 Mann Library Building, Ithaca, NY 14853, USA.

e-mail: jr286@cornell.edu
Next generation DNA sequencing technologies are driving increasingly rapid, affordable and high resolution analyses of plant transcriptomes through sequencing of their associated cDNA (complementary DNA) populations; an analytical platform commonly referred to as RNA-sequencing (RNA-seq). Since entering the arena of whole genome profiling technologies only a few years ago, RNA-seq has proven itself to be a powerful tool with a remarkably diverse range of applications, from detailed studies of biological processes at the cell type-specific level, to providing insights into fundamental questions in plant biology on an evolutionary time scale. Applications include generating genomic data for heretofore unsequenced species, thus expanding the boundaries of what had been considered "model organisms," elucidating structural and regulatory gene networks, revealing how plants respond to developmental cues and their environment, allowing a better understanding of the relationships between genes and their products, and uniting the "omics" fields of transcriptomics, proteomics, and metabolomics into a now common systems biology paradigm. We provide an overview of the breadth of such studies and summarize the range of RNA-seq protocols that have been developed to address questions spanning cell type-specific-based transcriptomics, transcript secondary structure and gene mapping.

Keywords: RNA-seq, plant transcriptome, transcriptomics, systems biology, next generation sequencing

\section{INTRODUCTION}

Next generation sequencing (NGS) is underpinning an ongoing revolution in the life sciences and it is now difficult to identify areas of biology that are not already being profoundly affected by the massive amounts of high quality DNA sequence information that has been generated cost-effectively and efficiently, thanks to the rapid advancement of sequencing technologies. Plant biology is naturally no exception to this revolution; indeed the ease of genetic analyses in many plant species and the value of crop species have made plant science an especially fertile area for many of the "omics" technologies. Plant scientists are rapidly moving on from a decade where the first genome sequence of a plant, that of Arabidopsis thaliana (The Arabidopsis Genome Initiative, 2000), provided the major impetus for monumental forays into plant molecular investigations, to the present day where the growing number of sequenced plant genomes ${ }^{1}$ is driving biological and evolutionary discovery across the plant taxonomic range.

In parallel with this explosion of genome sequence information, NGS has changed the scope and scale of transcriptome analysis and gene expression studies. RNA-sequencing (RNA-seq) technologies, which apply the principles of NGS to the complementary DNAs (cDNAs) derived from transcript populations, were first used to study plants only a few years ago (Weber et al., 2007) and now provide ready access to high resolution transcriptome information to an extent that was once unimaginable. This is

${ }^{1}$ http://genomevolution.org/wiki/index.php/Sequenced_plant_genomes exemplified by the $1 \mathrm{KP}$ project ${ }^{2}$, which aims to sequence the transcriptomes of 1,000 plant species, and is just one of many current initiatives that are radically expanding the breadth and depth of our understanding of plant gene expression and evolution. Due to its accuracy and the ease of meaningful comparisons of samples not necessarily generated together, or even as part of the same experiment, RNA-seq is replacing other methods of quantifying transcript expression, including cDNA- and expressed sequenced tag (EST)-based microarray platforms (Alba et al., 2004), as it overcomes many of their limitations (for an overview of RNA-seq technologies and comparisons with previous transcript detection technologies, see Wang et al., 2009). For example, RNA-seq approaches have an open architecture, meaning that they are not restricted to detecting only those transcripts that are represented on microarrays, and also exhibit more extreme upper and lower limits of detection, which allows more accurate quantification of differential transcript expression, as well as the identification of low-abundance transcripts. Furthermore, no previous genome sequence knowledge is necessary, as RNA-seq data sets themselves can be used to create sequence assemblies for subsequent mapping of RNA-seq reads, along with the potential for detecting exon/exon boundaries, alternative splicing and novel transcribed regions in a single sequencing run. However, despite these advantages, RNA-seq profiling platforms come with their own practical challenges. Existing RNA-seq techniques generate large numbers of relatively short reads for a particular transcript and so the

${ }^{2}$ http://www.onekp.com 
accurate assembly and annotation of the huge amounts of data generated by each run is still computationally difficult (Schliesky et al., 2012). Moreover, various biases can be introduced during the RNA fragmentation step prior to library construction, and cDNA fragmentation enriches the reads mapping the $3^{\prime}$ end of transcripts (Wang et al., 2009).

Nonetheless, RNA-seq has emerged as a remarkable enabling technology that is increasingly being adopted by plant researchers from a broad range of disciplines and examples of some of the associated applications and fields of research are presented in this review.

\section{IMPROVING GENOME ANNOTATION WITH TRANSCRIPTOMIC DATA}

More than a decade after the publication of the first draft of the A. thaliana genome sequence (The Arabidopsis Genome Initiative, 2000) its annotation continues to be improved. Large amounts of Sanger sequencing-generated EST data provided the initial basis for gene identification and expression profiling (Zhu et al., 2003), but such data are expensive and time consuming to generate, are inherently biased against low-abundance transcripts and are typically enriched in transcript termini (Filichkin et al., 2010). RNA-seq circumvents these limitations and provides accurate resolution of splice junctions and alternative splicing events. For example, a survey of the Arabidopsis transcriptome using single-base resolution Illumina-generated reads identified thousands of novel alternatively spliced transcripts and indicated that at least $42 \%$ of intron-containing genes are alternatively spliced (Filichkin et al., 2010). This percentage is considerably higher than previous estimations and is even greater (61\%) when only the multiexonic genes are sampled (Marquez et al., 2012). Similarly, approximately $48 \%$ of rice (Oryza sativa) genes show alternative splicing patterns (Lu et al., 2010), although more species need to be analyzed to determine whether this proportion is common. Mining RNA-seq data in search of transcription start site (TSS) variation is also improving gene structure annotation and alternative TSSs have been detected in $\sim 10,000$ loci through analyses of full-length Arabidopsis and rice cDNAs (Tanaka et al., 2009). RNAseq analysis also helps elucidate full-length transcript sequences, as has been demonstrated in a study where $\sim 10 \%$ of the untranslated region (UTR) boundaries of rice genes could be extended (Lu et al., 2010).

An ideal genome annotation would identify both genes that show invariant transcript sequences and those that exhibit alternative splicing, and additionally link these events to specific spatial, temporal, developmental, and/or environmental cues. Efforts in this direction are already underway and, as an example, it has been reported that abiotic stress in Arabidopsis can increase or decrease the proportions of apparently unproductive isoforms for some key regulatory genes, supporting the hypothesis that alternative splicing is an important mechanism in the regulation of gene function (Filichkin et al., 2010).

For many heterozygous and out-crossing species, genome sequencing and annotation can only be considered complete once the breadth of intra-species polymorphism is also considered. The high quality reference genome of $A$. thaliana is based on the ecotype Columbia (Col-0). It has been reported that polymorphisms between different $A$. thaliana accessions is relatively high, with one single nucleotide polymorphism (SNP) every 200 bp (Ossowski et al., 2008). The complete re-sequencing of the transcriptomes and annotation of different accessions may thus help interpret the functional consequences of polymorphism (Gan et al., 2011). To this end, utilizing genomic and transcriptomic data for in silico gene prediction results in a more reliable annotated genome, with information on SNPs, insertion/deletions (indels), splice variants and expression variation. Furthermore, with its greater sensitivity, RNA-seq enables the detection of antisense transcripts and transcribed intergenic regions; topics that are discussed further in Section "Identifying and Characterizing Novel Non-Coding RNAs."

\section{GENERATING GENOMIC AND ENABLING PROTEOMIC RESOURCES FOR “NON-MODEL” SPECIES}

Despite the recent upsurge in published plant genome sequences, they still represent a very small fraction of plant taxonomic diversity and the availability of transcriptomic information based on Sanger sequence-derived ESTs is similarly sparse, rendering the study of "non-model" species challenging. The very large genomes often encountered in plants, frequently associated with high sequence repeat regions, makes de novo sequencing of the transcriptome an attractive alternative to generate genetic resources for species that are of considerable biological interest for reasons that relate to factors such as their evolutionary significance or economic importance. Examples of recent such initiatives include fern (Der et al., 2011), eucalyptus (Mizrachi et al., 2010), garlic (Sun et al., 2012), pea (Franssen et al., 2011), chestnut (Barakat et al., 2009), chickpea (Garg et al., 2011), olive (Alagna et al., 2009), safflower (Lulin et al., 2012), and Japanese knotweed (Hao et al., 2011). The annotation of genes identified by de novo sequencing typically relies on identifying homologs, and ideally orthologs, in species with an annotated genome if no appropriate EST databases are available. An example of such annotation, using a pre-existing EST database associated with the species of interest, was reported for melon (Dai et al., 2011). Use of the annotated genome of a close-related species (e.g., Barrero et al., 2011) is preferable, but if none is available, the A. thaliana genome sequence is still widely regarded as the "gold standard" and can be extremely valuable to this end (e.g., Bräutigam et al., 2011). Further confirmation can then be sought by interrogating additional plant databases (e.g., Dassanayake et al., 2009; Edwards et al., 2012), although this depends on the standard of annotation and care should be taken that the database of interest is of high quality.

De novo RNA-seq to identify genetic polymorphisms also has great potential as a platform for molecular breeding, wherein multiple cultivars or close-related species with variations in traits of interest are sequenced and genetic variation is identified. This then allows the generation of molecular markers to facilitate progeny selection and molecular genetics research. As an example of this approach, the identification of 12,000 single sequence repeats (SSRs) in a single RNA-seq analysis of sesame (Zhang et al., 2012) increased the number of known SSRs from 80 to several thousand with, on average, one genic-SSR per $\sim 8 \mathrm{~kb}$. Similarly, Haseneyer et al. (2011) sampled the transcriptomes of five winter rye inbred lines to identify 5,234 SNPs, which were then incorporated in 
a high-throughput SNP genotyping array, further demonstrating the value of RNA-seq as a tool for advanced molecular breeding.

Another striking example of the value of RNA-seq as an enabling technology is its application to advance the field of proteomics. High-throughput mass spectrometry-based protein identification relies on the availability of an extensive DNA sequence database in order to match experimentally determined peptide masses with the theoretical proteome generated by computationally translating transcripts. Indeed, the lack of extensive plant DNA sequence information and related resources is likely a contributing factor in the relatively slow progress in the arena of plant proteomics compared with proteome studies of other organisms for which high quality sequence has long been available. Lopez-Casado etal. (2012) recently demonstrated that RNAseq-based transcriptome profiling can provide an effective data set for proteomic analysis of non-model organisms by de novo assembly of 454-based ESTs derived from the pollen of tomato (Solanum lycopersicum) and two wild relatives. Approximately the same number of proteins was identified when using either the RNA-seq-derived database, generated through a few 454 pyrosequencing runs, or a highly curated community database of tomato sequences generated over more than a decade. This suggests that RNA-seq will be invaluable in facilitating protein identification and that proteome studies need no longer be so taxonomically restricted.

\section{CHARACTERIZING TEMPORAL, SPATIAL, REGULATORY, AND EVOLUTIONARY TRANSCRIPTOME LANDSCAPES}

As with previous large-scale transcript profiling platforms, including microarrays, RNA-seq is increasingly being adopted to examine transcriptional dynamics during various aspects of plant growth and development. For example, an analysis of the transcriptome of grape (Vitis vinifera) berries during three stages of development identified $>6,500$ genes that were expressed in a stage-specific manner (Zenoni etal., 2010). Evidence of even greater transcriptomic complexity was provided by the detection of 210 and 97 genes that undergo alternative spicing in one or two stages, respectively. Similarly, Wang et al. (2012) analyzed the transcriptome of radish (Raphanus sativum) roots at two developmental stages and found $>21,000$ genes to be differentially expressed, including genes strongly linking root development with starch and sucrose metabolism and with phenylpropanoid biosynthesis. The radish genome has yet to be sequenced, but comparative sequence analysis of the radish RNAseq data and the Brassica rapa genome sequence lead to the discovery of 14,641 SSRs.

Most RNA-seq analyses target whole organs, or sets of organs, which inherently prevents the identification of cell or tissue type transcripts, and thus spatially coordinated structural and regulatory gene networks. Furthermore, transcripts that are expressed at extremely low levels, or that are specific to an uncommon cell type in a complex organ or tissue, may be diluted below the limit of detection. Accordingly, RNA-seq analysis of discrete tissues or cell types has the potential to both yield an important level of spatial information and substantially increase the depth of sequence coverage. As an example, Chen et al. (2010) detected more than 1,000 genes that are specifically or preferentially expressed in Arabidopsis male meiocytes that had been isolated by mechanically disrupting anthers with forceps and collecting the released meiocytes with a capillary pipette. However, acquiring tissue or cell-specific samples with any degree of precision and minimal contamination is often technically difficult, although several methods have been developed to facilitate this. For example, a cell type gene expression map of an Arabidopsis root was achieved by generating a set of transgenic Arabidopsis lines expressing green fluorescent protein (GFP) driven by various root cell type-specific promoters, digesting entire roots with cell wall degrading enzymes and fractionating the resulting protoplasts into distinct pools using an automated cell sorter (Birnbaum et al., 2003). The constituent root cell type-related transcriptomes were then analyzed using a microarray, providing a high resolution profile of the spatial variation in the root transcriptome. An alternative approach, which requires no prior genetic transformation or cell wall digestion, is laser capture microdissection (LCM), where a laser is used to excise and isolate samples from tissue sections with micron-scale resolution. This technique has been effectively used by plant researchers in conjunction with microarray analysis (Nakazono et al., 2003; Cai and Lashbrook, 2008; Agustí et al., 2009; Brooks et al., 2009; Matas et al., 2010). More recently, Matas et al. (2011) used LCM in combination with RNA-seq (454 pyrosequencing) analysis to profile the transcriptomes of the five principal tissues of the developing tomato fruit pericarp. Approximately 21,000 unigenes were identified, of which more than half showed ubiquitous expression, while other subsets showed clear cell type-specific expression patterns, providing insights into numerous aspects of fruit biology. A similar number of genes was identified in an LCM-based study of the ontogeny of maize (Zea mays) shoot apical meristems using RNA-seq coupled with Illumina-based NGS (Takacs et al., 2012). Interestingly, 59\% of the transcripts were detected in all the samples, comprising the apical domains along a developmental gradient from maize embryos to seedlings; a value that is very similar to the percentage of unigenes present in all tissues of the tomato fruit (57\%) reported by Matas et al. (2011), and the proportion of ubiquitously detected transcripts in the root cell sorting analysis (Birnbaum et al., 2003). RNA-seq profiling analyses of a number of mammalian tissues have also indicated a high proportion of ubiquitously expressed transcripts, which may indicate that this is a common feature of eukaryotes (Ramsköld et al., 2009).

In addition to studies focusing on transcriptional changes during development, RNA-seq has already shown itself to be a highly effective strategy to study plant responses and adaptations to abiotic and biotic stresses. For example, by analyzing RNA-seq data derived from sorghum (Sorghum bicolor) plants treated with abscisic acid (ABA) or polyethylene glycol, in conjunction with published transcriptome analysis for Arabidopsis, maize, and rice, Dugas et al. (2011) discovered > 50 previously unknown droughtresponsive genes. Similarly, RNA-seq was used to reveal massive changes in metabolism and cellular physiology of the green alga Chlamydomonas reinhardtii when the cells become deprived of sulfur, and to suggest molecular mechanisms that are used to tolerate sulfur deprivation (González-Ballester et al., 2010). Equivalent high resolution gene expression information has also resulted from 
studies of plant responses to pathogens and the complexities of the metabolic pathways associated with plant defense mechanisms. Published examples to date include a transcriptomic analysis of the infection of sorghum by the fungus Bipolaris sorghicola (Mizuno etal., 2012) and an investigation into the defense mechanisms of soybean that provide resistance to Xanthomonas axonopodis, by comparing resistant and susceptible near-isogenic lines (Kim et al., 2011).

As well as its applications to study spatial and temporal transcriptome dynamics, RNA-seq is also a potentially valuable tool to advance studies of plant evolution and polyploidy. As an illustration, a comparison of the leaf transcriptome of an allopolyploid relative of soybean with those of the two species that contributed to its homoelogous genome, allowed the determination of the contribution of the different genomes to the transcriptome (Ilut et al., 2012). Another study analyzed the transcriptome of nine distinct tissues of three species of the Poaceae family (Davidson etal., 2012) to determine whether orthologous genes from these three species exhibit the same expression patterns. Knowledge of parental imprinting has also been substantially advanced by deep transcriptome surveys. Despite the discovery of genetic imprinting in maize 40 years ago, only seven maize imprinted genes were reported before large-scale transcriptomic sequencing was applied to maize endosperm, leading to the discovery of 179 imprinted genes and 38 imprinted long ncRNAs (lncRNAs; Zhang etal., 2011). Studies of the embryo and endosperm of Arabidopsis and rice similarly increased the numbers of known imprinted genes and showed that imprinting is primarily endosperm-specific (Gehring et al., 2011; Hsieh et al., 2011; Luo et al., 2011).

We note that the studies cited in this section highlight the tremendous diversity of RNA-seq applications and the breadth of research fields in which it is being adopted, and the purpose is to provide examples, rather than a comprehensive list.

\section{IDENTIFYING AND CHARACTERIZING NOVEL NON-CODING RNAs}

Small RNAs (sRNAs) play important roles in gene posttranscriptional regulation (Baulcombe, 2004; Zamore and Haley, 2005) and there is great interest in developing techniques to comprehensively profile sRNA populations. In silico analysis provides a rapid way to identify putative sRNA genes (Chen et al., 2003, 2011; Hirsch et al., 2006) but RNA-seq technology represents an excellent means for sRNA discovery and validation. Indeed, deep sequencing of sRNAs has already been extensively used to find new sRNAs and especially microRNAs (miRNAs; Lu et al., 2005; Moxon et al., 2008; Szittya et al., 2008; Pantaleo et al., 2010; Song et al., 2010; Ferreira et al., 2012, Xia et al., 2012).

Characterization of miRNAs regulatory functions is likely to be facilitated by determining tissue-specific expression pattern, as shown by Breakfield et al. (2012) where RNA-seq was used to identify sRNAs from five Arabidopsis root tissues. Some sRNAs were expressed in all five tissues while others were tissue-specific, and some fluctuations in miRNA expression were also observed across developmental zones. In addition, growing numbers of RNA-seq studies are revealing the spatial and temporal differential expression of sRNAs in plant organs (Hirsch et al., 2006;
Moxon et al., 2008; Pantaleo et al., 2010; Calviño et al., 2011). The availability of high-throughput RNA-seq data allowed Yang et al. (2012) to mine these databases and discover that $\sim 12 \%$ of 354 high-confidence miRNA binding sites identified in Arabidopsis are affected by alternative splicing. The frequency of alternative slicing at miRNA binding sites is significantly higher than that at other regions, suggesting that alternative splicing is a significant regulatory mechanism. Small ncRNAs (sncRNAs) are also implicated in abiotic stresses and many miRNAs and other sRNAs have been shown to be differentially expressed under phosphate starvation in Arabidopsis roots and shoots (Hsieh et al., 2009), or under cold conditions (Zhang etal., 2009). The large amounts of data easily generated by RNA-seq also enable comparisons of sRNA populations between species, as demonstrated by Moxon et al. (2008), who found two tomato miRNAs that were previously believed to be specific to Arabidopsis or moss. In contrast to the numerous studies of plant sRNAs, far less is known about lncRNAs (>200 nt), especially in plants, and few plant lncRNAs have been characterized to date (Au et al., 2011; Kim and Sung, 2011; Zhu and Wang, 2012). Those that have been identified did not involve RNA-seq and so this represents an area with great potential for discovery.

Finally, sRNAs have been recently characterized in the context of association with epigenome modifications, including cytosine methylation of genomic DNA. While the majority of such work has involved animal systems, whole genome methylation analysis of epigenetic variation in Arabidopsis and rice embryo development, combined with sRNA analysis of the same tissues, confirmed a link between demethylation of certain gene promoters and associated miniature inverted repeats with changes in sRNA abundance (Cokus et al., 2008; Lister et al., 2008; Zemach et al., 2010). Interestingly, while promoter demethylation of tomato ripening genes was also recently described, it did not occur in conjunction with notable changes in sRNAs (Zhong et al., 2013). Genome-scale analyses of gene and sRNA expression via RNAseq, combined with whole genome methylation analyses are now facilitating the exploration of epigenomes in ways that could not have been considered prior to these high-throughput sequencing technologies.

\section{FROM CO-EXPRESSION NETWORKS TO INTEGRATIVE DATA ANALYSIS}

Sequencing whole transcriptomes provides a high degree of detail, but deriving useful biological information from a long list of expressed genes is typically not trivial. One approach to using such information to develop and refine hypotheses is to construct networks of co-expressed genes and to use gene ontology (GO) information to help highlight important gene candidates as critical components of functional networks. Many such "guilt-byassociation" gene co-expression networks have been constructed based on microarray data (Manfield et al., 2006; Mao et al., 2009; Childs et al., 2011; Tohge and Fernie, 2012) and are now being more widely adopted to evaluate RNA-seq data (Dugas et al., 2011; Iancu etal., 2012; Li etal., 2012). Indeed, the broad dynamic range of transcript level detection allowed by RNA-seq profiling, and particularly the detection of low-abundance transcripts, facilitates meaningful discrimination between different 


\section{cDNA synthesis}
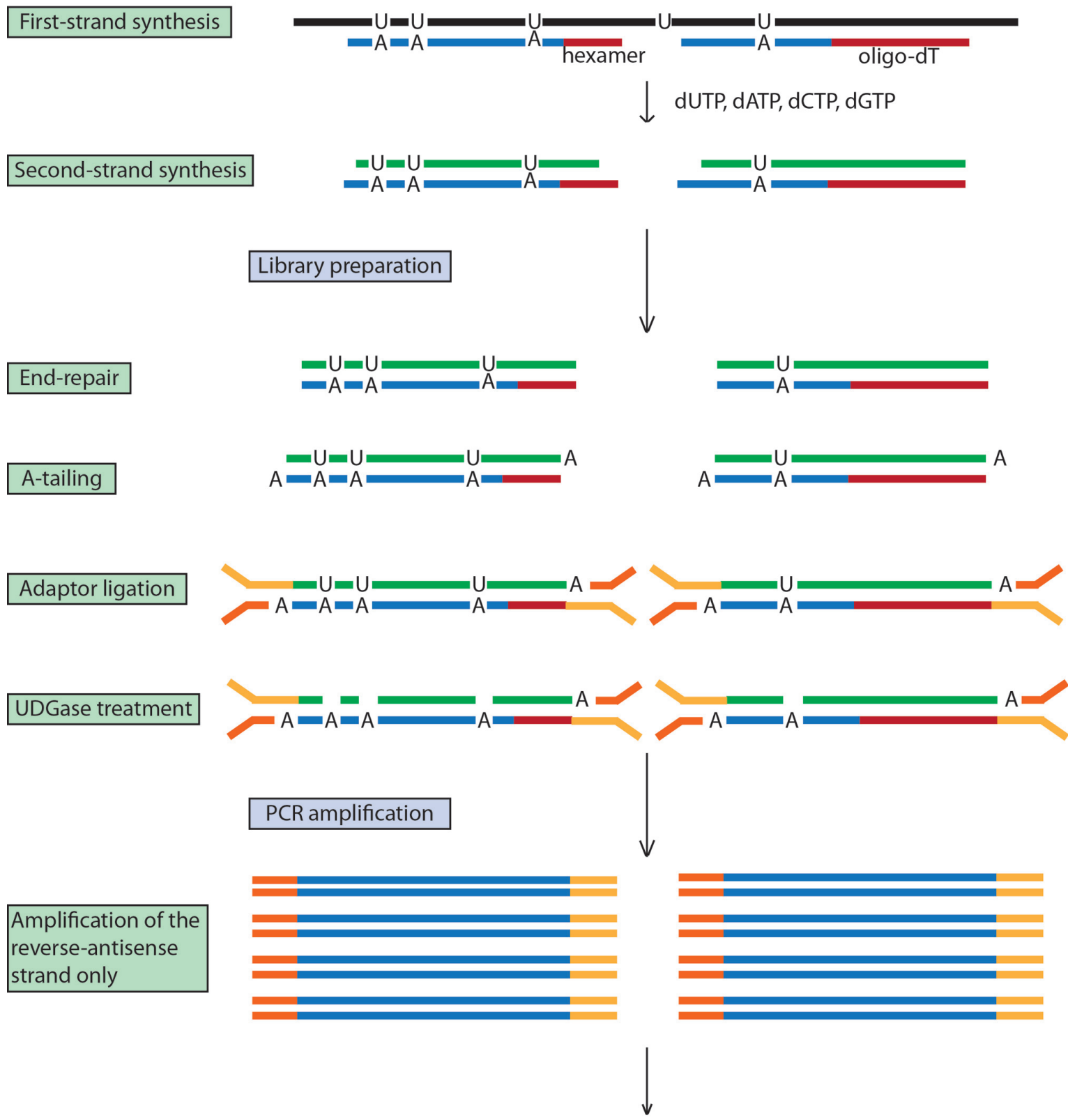

Sequencing

FIGURE 1 | dUTP-based strand-specific RNA-seq. Strand-specificity is achieved by the second-strand cDNA incorporating dUTPs instead of dTTPs. Digestion of dUTPs by uracil-DNA glycosylase (UGDase) prevents this strand from being PCR amplified, conferring single-strand specificity.

strengths of association in correlation analyses (Iancu et al., 2012). The correlations between different genes forming the expression network are therefore more robust and the overall expression network quality is generally superior to that generated using microarrays.

Gene ontology enrichment analysis of RNA-seq data often illustrates the complexity of interacting pathways. For example, in a study of abiotic stress responses in maize, transcripts associated with numerous GO classifications were affected by drought treatment, including the categories "carbohydrate metabolic process," "response to oxidative stress," and "cell division," among others (Kakumanu et al., 2012). The authors also showed that variations in GO term representation between organs can also provide valuable information and specifically, the drought-treated fertilized 


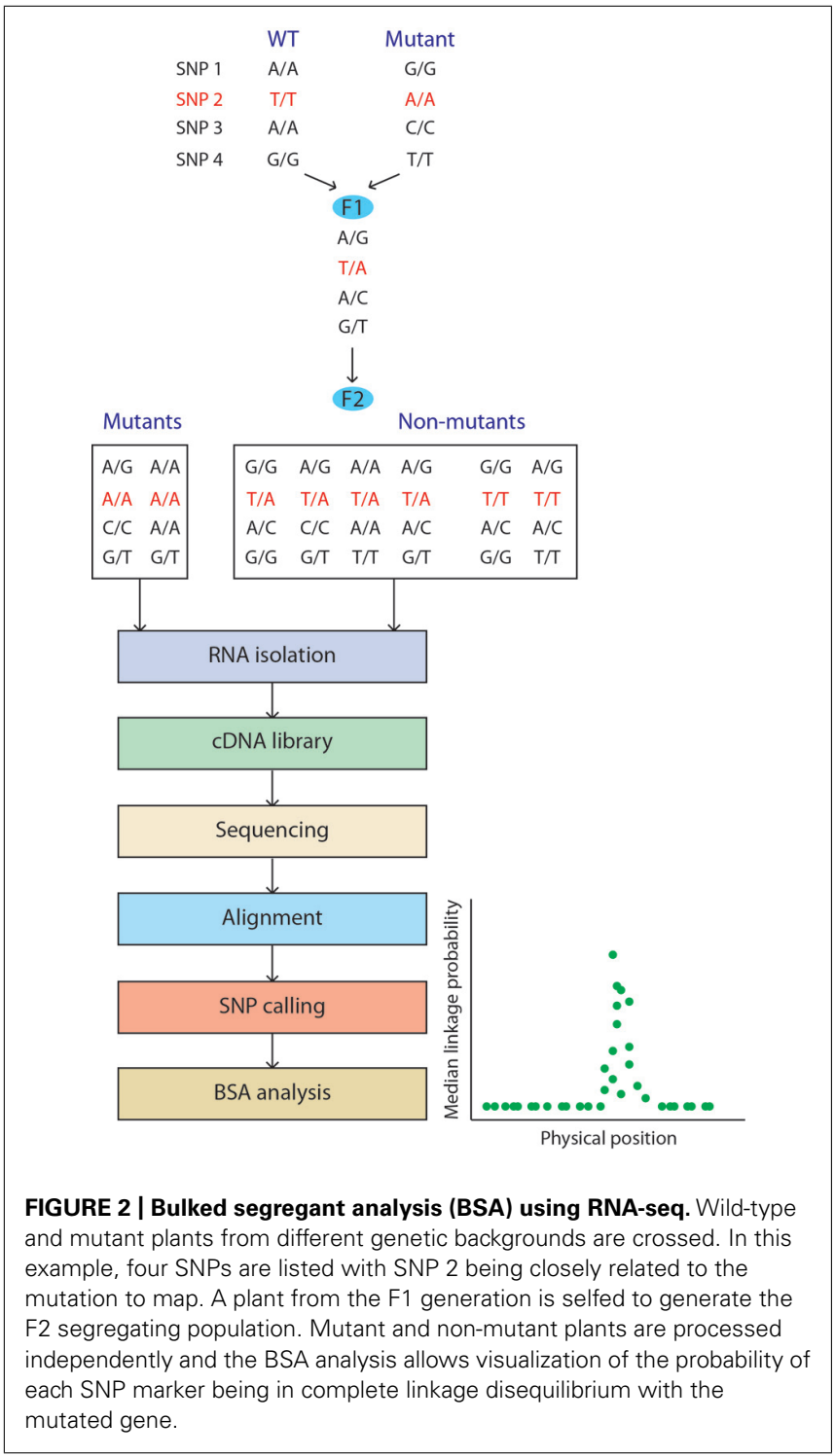

maize ovary exhibits a massive decrease of mRNAs involved in cell division and cell cycle, which could be the direct cause of the previously observed embryo abortion under drought conditions.

Functional networks can be made more robust by integrating multiple data types and various studies have coupled RNA-seq with proteomics and/or metabolomics, characterizing the apparent downstream consequences of transcript level variation. An example of such a "systems" study involved a comparative analysis of the transcriptome, proteome, and targeted metabolome of soybean seeds from transgenic lines with suppressed expression of the storage proteins glycinin and conglycinin (Schmidt et al., 2011). This study showed no direct correlation between the levels of transcripts, proteins, and metabolites. Conversely, a significant correlation was found between the high expression of fatty acid synthesis genes and the high oil content in oil palm mesocarp (Bourgis etal., 2011). These studies further demonstrate the value of characterizing biological processes from multiple "omics" perspectives, each of which can provide insights

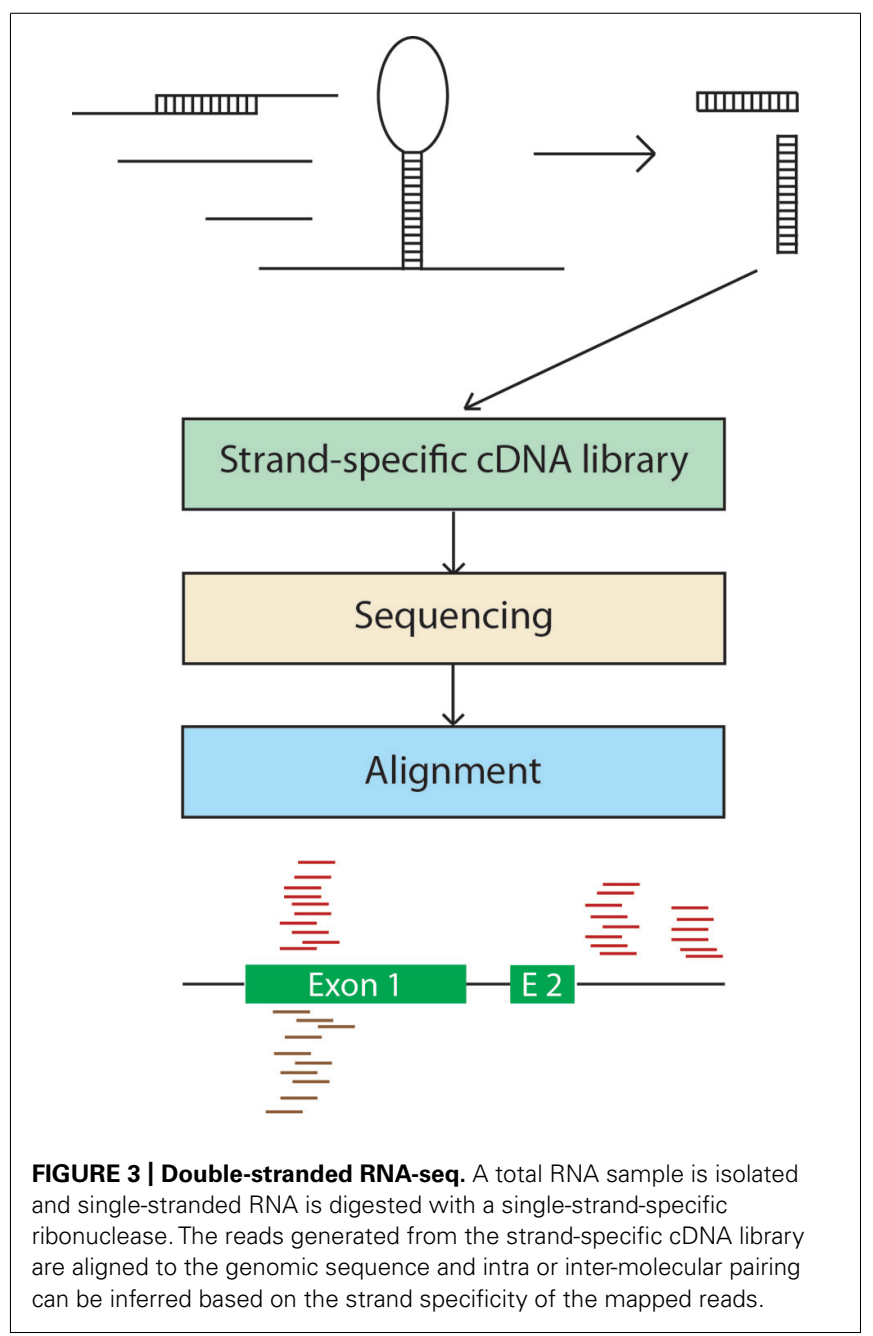

into different regulatory mechanisms. Surveying the metabolome and transcriptome in parallel can also help identify candidate genes involved in complex metabolic pathways. For example, Desgagné-Penix etal. (2012) took advantage of several opium poppy (Papaver somniferum) cultivars with known differential levels of benzylisoquinoline alkaloids (BIAs) and used a combination of RNA-seq and mass spectrometry to pinpoint key regulatory steps of the almost completely defined morphine biosynthetic pathway, leading to the discovery of candidate genes implicated in BIA metabolism.

These examples show that the integration of transcriptomics, proteomics, and metabolomics can expose complex biological and biochemical interactions, paving the way to elucidate relationships between genotype and phenotype. Even greater resolution can be achieved by targeting tissues instead of whole organs (Rogers et al., 2012).

\section{A GROWING PORTFOLIO OF RNA-seq ANALYTICAL STRATEGIES}

RNA-seq technologies can be adapted to answer-specific biological questions. Four different adaptations or applications are described here. 


\section{STRAND-SPECIFIC RNA-seq}

Standard RNA-seq methods do not discriminate between the DNA strands on which the RNAs are encoded. However, the ability to map a transcript to its specific coding strand is desirable as it improves transcript mapping accuracy by identifying non-coding antisense transcripts that may be involved in regulation at the messenger or at the chromatin levels (Ponting et al., 2009; Liu et al., 2010), helps determine the relative expression level of two genes on opposite DNA strands as well as their exact length, and allows the identification of the transcribed strand of ncRNAs. Levin et al. (2010) compared seven library construction methods to enable strand-specific RNA-seq analysis and overall, a dUTP method (Parkhomchuk etal., 2009) was the most accurate and has the advantage of being compatible with paired-end sequencing. This method has been successfully applied to plant RNA-seq with adaptations rendering it low-cost and high-throughput (Wang et al., 2011; Zhong et al., 2011). In short, the first cDNA strand is synthesized with dNTP while dUTP is incorporated in the second cDNA strand. After end repair, A-tailing and adaptor ligation, the dUTP-containing strand is digested and the remaining strand is PCR-amplified conferring strand specificity (Figure 1). As an example of the value of strand information, a study of tomato gene expression showed that while the majority of genes in the tissues analyzed had effectively the same expression profiles when analyzed by either double-stranded or strand-specific RNA-seq, approximately $5 \%$ of transcripts were associated with misleading results when assayed by double-stranded RNA-seq (dsRNA-seq) alone (Zhong et al., 2011).

\section{BULKED SEGREGANT RNA-seq}

Liu et al. (2012) demonstrated the application of RNA-seq for bulked segregant analysis (BSA) by mapping the maize mutant gene gl3. Transcriptome profiling is applied to a pool of two samples generated by mixing a bulk of mutant and wild-type (WT) plants (Figure 2). The mapping of the mutated gene is based on genetic linkage where linkage disequilibrium between markers and the causal gene is determined by quantifying the allelic frequencies between the two samples, giving the map position of the gene responsible for the mutant phenotype. Fine mapping of the mutated gene is facilitated by the RNA-seq data as its expression will often be down-regulated compared to the WT pool. Additionally, the SNPs linked to the mutated gene can be used for chromosome walking. Using RNA-seq for this purpose has therefore numerous advantages: (i) having a reference genome is not a prerequisite as de novo assembly of the transcriptome based on the RNA-seq data is sufficient; (ii) markers can be generated from the experimental data; and (iii) differential expression profiles between the mutant and the WT are generated at no extra cost. Furthermore, this approach can be modified to perform genomewide association (GWAS) studies, accelerating breeding initiatives by providing markers targeting both genetic sequence (e.g., SNPs) and gene expression, using them to identify the genomic regions associated with the traits of interest (Harper et al., 2012).

\section{DOUBLE-STRANDED RNA-seq}

Secondary structures of RNAs are central to their function, maturation, and regulation; however, little is known about the

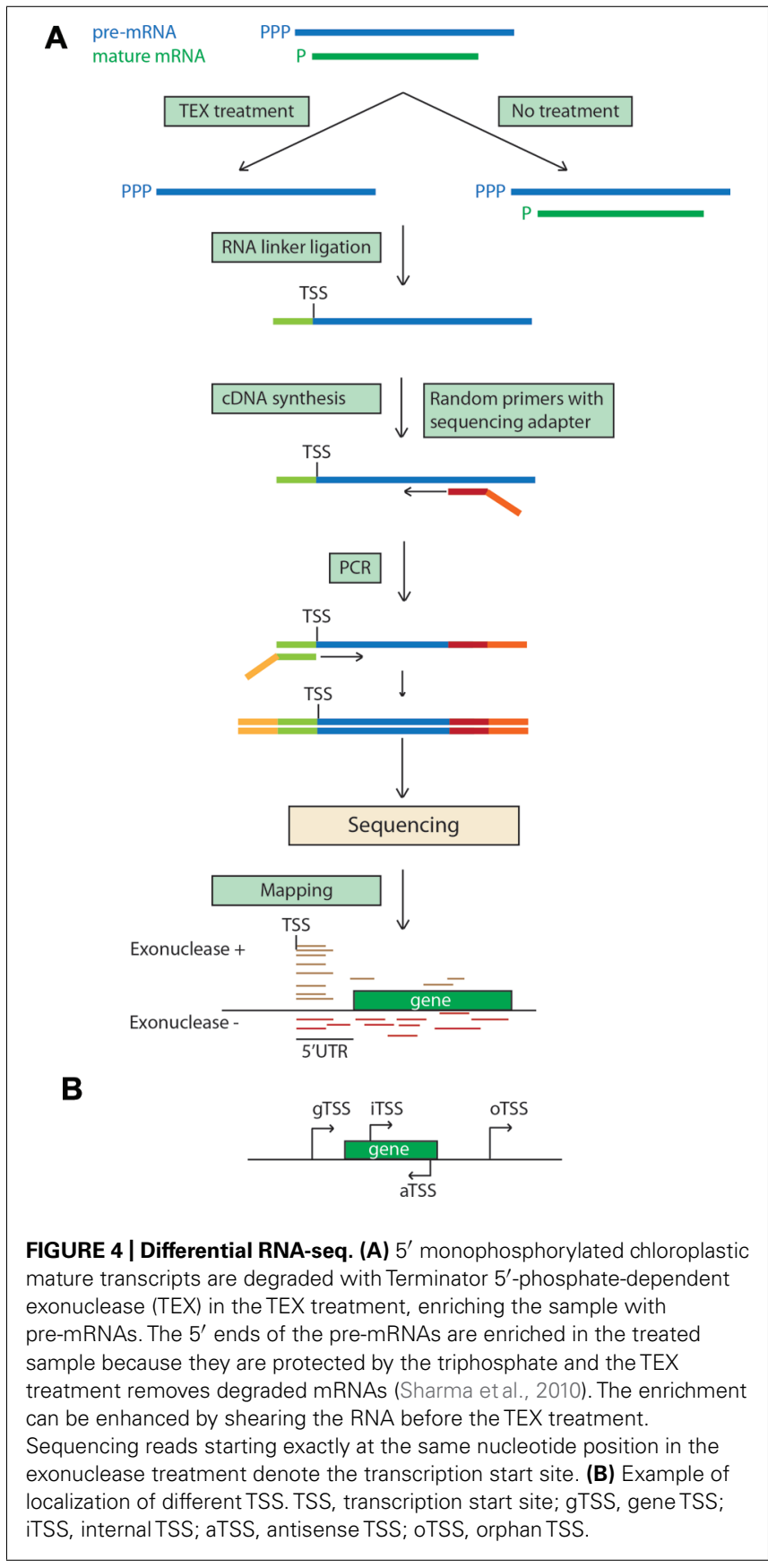

double-stranded features of most RNAs. Zheng et al. (2010) reported an experimental strategy to survey RNA secondary structures in an analysis of the double-stranded species of RNAs from Arabidopsis flower buds. Specifically, the authors sequenced only the double-stranded RNAs (dsRNAs) and the double-stranded segments of RNAs by digesting the single-stranded RNAs with a ribominus treatment prior to library construction (Figure 3 ). As expected, highly structured RNA classes (e.g., rRNA, tRNA, and snRNA) were highly represented in the reads but, interestingly, other regions of various mRNAs, including introns, exons, and $5^{\prime}$ and $3^{\prime}$ UTRs were also present, indicating the presence of mRNA secondary structures. Moreover, the double-stranded regions of 
the introns, $3^{\prime}$ and $5^{\prime}$ UTRs appeared to be conserved, suggesting a common function. Notably, certain regions of the genome appear to be responsible for producing more dsRNAs than others, with transposable elements representing nearly $60 \%$ of these "hotspots."

\section{DIFFERENTIAL RNA-seq}

Differential RNA-seq (dRNA-seq) is based on a comparison of a terminator exonuclease treated RNA sample with its non-treated counterpart (Figure 4). The treatment removes the processed transcripts by degrading $5^{\prime}$ monophosphate RNAs, which are characteristic of prokaryotic RNAs, and the primary unprocessed transcripts are not affected due to the presence of a $5^{\prime}$ triphosphate. By comparing the maps of the reads derived from each sample, TSSs of operons are identified. dRNA-seq was first used to examine the transcriptome of the human pathogen Helicobacter pylori (Sharma et al., 2010) and subsequently in studies of various prokaryotes, including the plant pathogen Pseudomonas syringae (Filiatrault et al., 2011). This method was used to map TSSs of barley chloroplastic RNAs (Zhelyazkova et al., 2012) and was possible as they have the same $5^{\prime}$ monophosphate structure as prokaryotic RNAs, reflecting the endosymbiotic origin of chloroplasts. Four categories of TSSs were identified in this study: gTSSs (g: gene) located within 750 nucleotides upstream of annotated genes (the majority of TSSs); iTSSs (i: internal) located within annotated genes and giving rise to sense transcripts; aTSSs (a: antisense) giving rise to antisense transcripts;

\section{REFERENCES}

Agustí, J., Merelo, P., Cercós, M., Tadeo, F. R., and Talón, M. (2009). Comparative transcriptional survey between laser-microdissected cells from laminar abscission zone and petiolar cortical tissue during ethylene-promoted abscission in citrus leaves. BMC Plant Biol. 9:127. doi: 10.1186/1471-22299-127

Alagna, F., D’Agostino, N., Torchia, L., Servili, M., Rao, R., Pietrella, M., et al. (2009). Comparative 454 pyrosequencing of transcripts from two olive genotypes during fruit development. BMC Genomics 10:399. doi: 10.1186/1471-2164-10-399

Alba, R., Fei, Z., Payton, P., Liu, Y., Moore, S. L., Debbie, P., et al. (2004). ESTs, cDNA microarrays, and gene expression profiling: tools for dissecting plant physiology and development. Plant J. 39, 697-714.

Au, P. C. K., Zhu, Q.-H., Dennis, E. S., and Wang, M.-B. (2011). Long noncoding RNA-mediated mechanisms independent of the RNAi pathway in animals and plants. RNA Biol. 8, 404-414.

Barakat, A., Diloreto, D. S., Zhang, Y., Smith, C., Baier, K., Powell, W. A., et al. (2009). Comparison of the transcriptomes of American chestnut (Castanea dentata) and Chinese chestnut (Castanea mollissima) in response to the chestnut blight infection. BMC Plant Biol. 9:51. doi: 10.1186/1471-2229-9-51

Barrero, R. A., Chapman, B., Yang, Y., Moolhuijzen, P., Keeble-Gagnère, G., Zhang, N., et al. (2011). De novo assembly of Euphorbia fischeriana root transcriptome identifies prostratin pathway related genes. $B M C$ Genomics 12:600. doi: 10.1186/14712164-12-600

Baulcombe, D. (2004). RNA silencing in plants. Nature 431, 356-363.

Birnbaum, K., Shasha, D. E., Wang, J. Y., Jung, J. W., Lambert, G. M., Galbraith, D. W., et al. (2003). A gene expression map of the Arabidopsis root. Science 302, 19561960.

Bourgis, F., Kilaru, A., Cao, X., NgandoEbongue, G.-F., Drira, N., Ohlrogge, J. B., et al. (2011). Comparative transcriptome and metabolite analysis of differ dramatically in carbon partitioning. Proc. Natl. Acad. Sci. U.S.A. 108, 12527-12532.

Bräutigam, A., Kajala, K., Wullenweber, J., Sommer, M., Gagneul, D., Weber, K. L., etal. (2011). An mRNA blueprint for C4 photosynthesis derived from comparative transcriptomics of closely related C3 and C4 species. Plant Physiol. 155, 142-156. oil palm and date palm mesocarp that

and oTSSs (o: orphan) located in intergenic regions. The analysis revealed that some individual transcriptional units of the chloroplastic operons can be transcribed individually as suggested by iTSSs and that $\sim 35 \%$ of chloroplastic genes have aTSSs or oTSSs, providing evidence of extensive ncRNAs synthesis in chloroplasts.

\section{CONCLUDING REMARKS}

RNA-sequencing is now well-established as a versatile platform with applications in an ever growing number of fields of plant biology research. Ongoing developments in sequencing technologies, such as increased read lengths, greater numbers of reads per run, and advanced computational tools to facilitate sequence assembly, analysis, and integration with orthogonal data sets will further accelerate the breadth and frequency of its adoption by plant scientists. An important issue that still needs to be addressed is the inherent bias introduced by the different steps of library construction and so the tantalizing prospect of direct RNA-seq (Ozsolak and Milos, 2011) has great promise in this regard.

\section{ACKNOWLEDGMENTS}

Funding to Jocelyn K. C. Rose and James J. Giovannoni for research in this area is provided by the NSF Plant Genome Research Program (DBI-0606595), and NSF EAGER award (Plant Genome Research Program) and the New York State Office of Science, Technology and Academic Research (NYSTAR).

Breakfield, N. W., Corcoran, D. L. Petricka, J. J., Shen, J., Sae-Seaw, J., Rubio-Somoza, I., et al. (2012). High-resolution experimental and computational profiling of tissuespecific known and novel miRNAs in Arabidopsis. Genome Res. 22, 163-176.

Brooks, L. III, Strable, J., Zhang, X., Ohtsu, K., Zhou, R., Sarkar, A., et al. (2009). Microdissection of shoot meristem functional domains. PLoS Genet. 5:e1000476. doi: 10.1371/journal.pgen.1000476

Cai, S., and Lashbrook, C. C. (2008). Stamen abscission zone transcriptome profiling reveals new candidates for abscission control: enhanced retention of floral organs in transgenic plants overexpressing Arabidopsis ZINC FINGER PROTEIN2. Plant Physiol. 146, 1305-1321.

Calviño, M., Bruggmann, R., and Messing, J. (2011). Characterization of the small RNA component of the transcriptome from grain and sweet sorghum stems. BMC Genomics 12:356. doi: 10.1186/1471-216412-356

Chen, C., Farmer, A. D., Langley, R. J., Mudge, J., Crow, J. A., May, G. D., et al. (2010). Meiosis-specific gene discovery in plants: RNA-Seq applied to isolated Arabidopsis male meiocytes. Plant Biol. 10, 280.
Chen, C.-J., Zhou, H., Chen, Y.-Q., Qu, L.-H., and Gautheret, D. (2011). Plant noncoding RNA gene discovery by "single-genome comparative genomics". Bioinformatics 17, 390-400.

Chen, C.-L., Liang, D., Zhou, H., Zhuo, M., Chen, Y.-Q., and Qu, L.-H. (2003). The high diversity of snoRNAs in plants: identification and comparative study of 120 snoRNA genes from Oryza sativa. Nucleic Acids Res. 15, 2601-2613.

Childs, K. L., Davidson, R. M., and Buell, C. R. (2011). Gene coexpression network analysis as a source of functional annotation for rice genes. PLoS ONE 6:e22196. doi: 10.1371/journal.pone.0022196

Cokus, S. J., Feng, S., Zhang, X., Chen, Z., Merriman, B., Haudenschild, C. D., etal. (2008). Shotgun bisulphite sequencing of the Arabidopsis genome reveals DNA methylation patterning. Nature 452, 215-219.

Dai, N., Cohen, S., Portnoy, V., Tzuri, G., Harel-Beja, R., Pompan-Lotan, M., et al. (2011). Metabolism of soluble sugars in developing melon fruit: a global transcriptional view of the metabolic transition to sucrose accumulation. Plant Mol. Biol. 76, 1-18.

Dassanayake, M., Haas, J. S., Bohnert, H. J., and Cheeseman, J. M. (2009). Shedding light on 
an extremophile lifestyle through transcriptomics. New Phytol. 183, 764-775.

Davidson, R. M., Gowda, M., Moghe, G., Lin, H., Vaillancourt, B., Shiu, S.H., et al. (2012). Comparative transcriptomics of three Poaceae species reveals patterns of gene expression evolution. Plant J. 71, 492-502.

Der, J. P., Barker, M. S., Wickett, N. J., Depamphilis, C. W., and Wolf, P. G. (2011). De novo characterization of the gametophyte transcriptome in bracken fern, Pteridium aquilinum. BMC Genomics 12:99. doi: 10.1186/1471-2164-12-99

Desgagné-Penix, I., Farrow, S. C., Cram, D., Nowak, J., and Facchini, P. J. (2012). Integration of deep transcript and targeted metabolite profiles for eight cultivars of opium poppy. Plant Mol. Biol. 79, 295-313.

Dugas, D. V., Monaco, M. K., Olsen, A., Klein, R. R., Kumari, S., Ware, D., et al. (2011). Functional annotation of the transcriptome of Sorghum bicolor in response to osmotic stress and abscisic acid. BMC Genomics 12:514. doi: 10.1186/1471-216412-514

Edwards, C. E., Parchman, T. L., and Weekley, C. W. (2012). Assembly, gene annotation and marker development using 454 floral transcriptome sequences in Ziziphus celata (Rhamnaceae), a highly endangered, florida endemic plant. DNA Res. 19, 1-9.

Ferreira, T. H., Gentile, A., Vilela, R. D., Costa, G. G. L., Dias, L. I., Endres, L., et al. (2012). microRNAs associated with drought response in the bioenergy crop sugarcane (Saccharum spp.). PLoS ONE 7:e46703. doi: 10.1371/journal.pone.0046703

Filiatrault, M. J., Stodghill, P. V., Myers, C. R., Bronstein, P. A., Butcher, B. G., Lam, H., et al. (2011). Genomewide identification of transcriptional start sites in the plant pathogen Pseudomonas syringae pv. tomato str. DC3000. PLOS ONE 6:e29335. doi: 10.1371/journal.pone.0029335

Filichkin, S. A., Priest, H. D., Givan, S. A., Shen, R., Bryant, D. W., Fox, S. E., et al. (2010). Genome-wide mapping of alternative splicing in Arabidopsis thaliana. Genome Res. 20, 45-58.

Franssen, S. U., Shrestha, R. P., Brautigam, A., Bornberg-Bauer, E., and Weber, A. P. M. (2011). Comprehensive transcriptome analysis of the highly complex Pisum sativum genome using next generation sequencing. BMC Genomics 12:227. doi: 10.1186/1471-216412-227

Gan, X., Stegle, O., Behr, J., Steffen, J. G., Drewe, P., Hildebrand, K. L., et al.
(2011). Multiple reference genomes and transcriptomes for Arabidopsis thaliana. Nature 477, 419-423.

Garg, R., Patel, R. K., Jhanwar, S., Priya, P., Bhattacharjee, A., Yadav, G., et al. (2011). Gene discovery and tissue-specific transcriptome analysis in chickpea with massively parallel pyrosequencing and web resource development. Plant Physiol. 156, 1661-1678.

Gehring, M., Missirian, V., and Henikoff, S. (2011). Genomic analysis of parent-of-origin allelic expression in Arabidopsis thaliana seeds. PLoS ONE 6:e23687. doi: 10.1371/journal.pone.0023687

González-Ballester, D., Casero, D., Cokus, S., Pellegrini, M., Merchant, S. S., and Grossman, A. R. (2010). RNA-seq analysis of sulfur-deprived Chlamydomonas cells reveals aspects of acclimation critical for cell survival. Plant Cell 22, 2058-2084.

Hao, D. C., Ge, G. B., Xiao, P. G., Zhang, Y. Y., and Yang, L. (2011). The first insight into the tissue specific taxus transcriptome via illumina second generation sequencing. PLoS ONE 6:e21220. doi: 10.1371/journal.pone.0021220

Harper, A. L., Trick, M., Higgins, J., Fraser, F., Clissold, L., Wells, R., et al. (2012). Associative transcriptomics of traits in the polyploid crop species Brassica napus. Nat. Biotechnol. 30, 798-802.

Haseneyer, G., Schmutzer, T., Seidel, M., Zhou, R., Mascher, M., Schön, C.-C. et al. (2011). From RNA-seq to largescale genotyping: genomics resources for rye (Secale cereale L.). BMC Plant Biol. 11:131. doi: 10.1186/1471-222911-131

Hirsch, J., Lefort, V., Vankersschaver, M., Boualem, A., Lucas, A., Thermes, C., et al. (2006). Characterization of 43 non-protein-coding mRNA genes in Arabidopsis, including the MIR162aderived transcripts. Plant Physiol. 140, 1192-1204.

Hsieh, L.-C., Lin, S.-I., Shih, A. C.-C., Chen, J.-W., Lin, W.-Y., Tseng, C.Y., et al. (2009). Uncovering small RNA-mediated responses to phosphate deficiency in Arabidopsis by deep sequencing. Plant Physiol. 151, 2120-2132.

Hsieh, T.-F., Shin, J., Uzawa, R., Silva, P., Cohen, S., Bauer, M. J., et al. (2011). Regulation of imprinted gene expression in Arabidopsis endosperm. Proc. Natl. Acad. Sci. U.S.A. 108, 1755-1762.

Iancu, O. D., Kawane, S., Bottomly, D., Searles, R., Hitzemann, R., and McWeeney, S. (2012). Utilizing RNAseq data for de-novo coexpression network inference. Bioinformatics 28 , 1592-1597.

Ilut, D. C., Coate, J. E., Luciano, A. K., Owens, T. G., May, G. D., Farmer, A., et al. (2012). A comparative transcriptomic study of an allotetraploid and its diploid progenitors illustrates the unique advantages and challenges of RNA-seq in plant species. Am. J. Bot. 99, 383-396.

Kakumanu, A., Ambavaram, M. M. R., Klumas, C., Krishnan, A., Batlang, U., Myers, E., et al. (2012). Effects of drought on gene expression in maize reproductive and leaf meristem tissue revealed by RNA-seq. Plant Physiol. 160, 846-867.

Kim, E.-D., and Sung, S. (2011). Long noncoding RNA: unveiling hidden layer of gene regulatory networks. Trends Plant Sci. 17, 16-21.

Kim, K. H., Kang, Y. J., Kim, D. H., Yoon, M. Y., Moon, J. K., Kim, M Y., et al. (2011). RNA-seq analysis of a soybean near-isogenic line carrying bacterial leaf pustule-resistant and -susceptible alleles. DNA Res. 18, 483-497.

Levin, J. Z., Yassour, M., Adiconis, X., Nusbaum, C., Thompson, D. A., Friedman, N., et al. (2010). Comprehensive comparative analysis of strand-specific RNA sequencing methods. Nat. Methods 7, 709-715.

Li, W., Dai, C., Liu, C.-C., and Zhou, X. J. (2012). Algorithm to identify frequent coupled modules from twolayered network series: application to study transcription and splicing coupling. J. Comput. Biol. 19, 710-730.

Lister, R., O’Malley, R. C., TontiFilippini, J., Gregory, B. D., Berry, C. C., Millar, A. H., etal. (2008) Highly integrated single-base resolution maps of the epigenome in Arabidopsis. Cell 133, 523-536.

Liu, F., Marquardt, S., Lister, C., Swiezewski, S., and Dean, C. (2010). Targeted $3^{\prime}$ processing of antisense transcripts triggers Arabidopsis FLC chromatin silencing. Science 327, 94-97.

Liu, S., Yeh, C.-T., Tang, H. M., Nettleton, D., and Schnable, P. S. (2012). Gene mapping via bulked segregant RNA-seq (BSR-Seq). PLoS ONE 7:e36406. doi: 10.1371/journal.pone.0036406

Lopez-Casado, G., Covey, P. A., Bedinger, P. A., Mueller, L. A., Thannhauser, T. W., Zhang, S., et al. (2012). Enabling proteomic studies with RNA-seq: the proteome of tomato pollen as a test case. Proteomics 12, 761-774.

Lu, C., Singh Tej, S., Luo, S., Haudenschild, C. D., Meyers, B. C., and Green, P. J. (2005). Elucidation of the small RNA component of the transcriptome. Science 309, 1567-1569.

Lu, T., Lu, G., Fan, D., Zhu, C., Li, W., Zhao, Q., etal. (2010). Function annotation of the rice transcriptome at single-nucleotide resolution by RNA-seq. Genome Res. 20, 12381249.

Lulin, H., Xiao, Y., Pei, S., Wen, T., and Shangqin, H. (2012). The first illumina-based de novo transcriptome sequencing and analysis of safflower flowers. PLoS ONE 7:e38653. doi: 10.1371/journal.pone.0038653

Luo, M., Taylor, J. M., Spriggs, A., Zhang, H., Wu, X., Russel, S., et al. (2011). A genome-wide survey of imprinted genes in rice seeds reveals imprinting primarily occurs in the endosperm. PLoS Genet. 7:e1002125. doi: 10.1371/journal.pgen.1002125

Manfield, I. W., Jen, C.-H., Pinney, J. W., Michalopoulos, I., Bradford, J. R., Gilmartin, P. M., et al. (2006). Arabidopsis co-expression tool (ACT): web server tools for microarray-based gene expression analysis. Nucleic Acids Res. 34, 504-509.

Mao, L., Van Hemert, J. L., Dash, S., and Dickerson, J. A. (2009). Arabidopsis gene co-expression network and its functional modules. BMC Bioinformatics 10:346. doi: 10.1186/14712105-10-346

Marquez, Y., Brown, J. W. S., Simpson, C., Barta, A., and Kalyna, M. (2012). Transcriptome survey reveals increased complexity of the alternative splicing landscape in Arabidopsis. Genome Res. 22, 1184-1195.

Matas, A. J., Agustí, J., Tadeo, F. R., Talón, M., and Rose, J. K. C. (2010). Tissue specific transcriptome profiling of the citrus fruit epidermis and subepidermis using laser capture microdissection. J. Exp. Bot. 61, 3321-3330.

Matas, A. J., Yeats, T. H., Buda, G. J., Zheng, Y., Chatterjee, S., Tohge, T., etal. (2011). Tissueand cell-type specific transcriptome profiling of expanding tomato fruit provides insights into metabolic and regulatory specialization and cuticle formation. Plant Cell 23, 3893-3910.

Mizrachi, E., Hefer, C. A., Ranik, M., Joubert, F., and Myburg, A. A. (2010). De novo assembled expressed gene catalog of a fast-growing eucalyptus tree produced by Illumina mRNASeq. BMC Genomics 11:681. doi: 10.1186/1471-2164-11-681

Mizuno, H., Kawahigashi, H., Kawahara, Y., Kanamori, H., Ogata, J., Minami, H., et al. (2012). Global transcriptome analysis reveals distinct expression among 
duplicated genes during sorghumBipolaris sorghicola interaction. BMC Plant Biol. 12:121. doi: 10.1186/1471-2229-12-121

Moxon, S., Jing, R., Szittya, G., Schwach, F., Rusholme Pilcher, R. L., Moulton, V., et al. (2008). Deep sequencing of tomato short RNAs identifies microRNAs targeting genes involved in fruit ripening. Genome Res. 18, 1602-1609.

Nakazono, M., Qiu, F., Borsuk, L. A., and Schnable, P. S. (2003). Lasercapture microdissection, a tool for the global analysis of gene expression in specific plant cell types: identification of genes expressed differentially in epidermal cells or vascular tissue of maize. Plant Cell 15, 583-596.

Ossowski, S., Schneeberger, K., Clark, R. M., Lanz, C., Warthmann, N., and Weigel, D. (2008). Sequencing of natural strains of Arabidopsis thaliana with short reads. Genome Res. 18, 2024-2033.

Ozsolak, F., and Milos, P. M. (2011). RNA sequencing: advances, challenges and opportunities. Nat. Rev. Genet. 12, 87-98.

Pantaleo, V., Szittya, G., Moxon, S., Miozzi, L., Moulton, V., Dalmay, T., et al. (2010). Identification of grapevine microRNAs and their targets using high-throughput sequencing and degradome analysis. Plant $J$. 62,960-976.

Parkhomchuk, D., Borodina, T., Amstislavskiy, V., Banaru, M., Hallen, L., Krobitsch, S., et al. (2009). Transcriptome analysis by strand-specific sequencing of complementary DNA. Nucleic Acids Res. 37, e123.

Ponting, C. P., Oliver, P. L., and Reik, W. (2009). Evolution and functions of long noncoding RNAs. Cell 136, 629-641.

Ramsköld, D., Wang, E. T., Burge, C. B., and Sandberg, R. (2009). An abundance of ubiquitously expressed genes revealed by tissue transcriptome sequence data. PLoS Comput. Biol. 5:e1000598. doi: 10.1371/journal.pcbi. 1000598

Rogers, E. D., Jackson, T., Moussaieff, A., Aharoni, A., and Benfey, P. N. (2012). Cell type-specific transcriptional profiling: implications for metabolite profiling. Plant J. 70, 5-17.

Schliesky, S., Gowik, U., Weber, A. P. M., and Bräutigam, A. (2012). RNA-seq assembly -are we there yet? Front. Plant Sci. 3:220. doi: 10.3389/fpls.2012.00220
Schmidt, M. A., Barbazuk, W. B., Sandford, M., May, G., Song, Z., Zhou, W., et al. (2011). Silencing of soybean seed storage proteins results in a rebalanced protein composition preserving seed protein content without major collateral changes in the metabolome and transcriptome. Plant Physiol. 156, 330-345.

Sharma, C. M., Hoffmann, S., Darfeuille, F., Reignier, J., Findeiß, S. Sittka, A., et al. (2010). The primary transcriptome of the major human pathogen Helicobacter pylori. Nature 464, 250-255.

Song, C., Wang, C., Zhang, C., Korir, N. K., Yu, H., Ma, Z., et al. (2010) Deep sequencing discovery of novel and conserved microRNAs in trifoliate orange (Citrus trifoliata). BMC Genomics 11:431. doi: 10.1186/14712164-11-431

Sun, X., Zhou, S., Meng, F., and Liu, S. (2012). De novo assembly and characterization of the garlic (Allium sativum) bud transcriptome by Illumina sequencing. Plant Cell 31 , 1823-1828.

Szittya, G., Moxon, S., Santos, D. M., Jing, R., Fevereiro, M. P. S., Moulton, V., et al. (2008). High-throughput sequencing of Medicago truncatula short RNAs identifies eight new miRNA families. BMC Genomics 9:593. doi: 10.1186/1471-2164-9-593

Takacs, E. M., Li, J., Du, C., Ponnala, L., Janick-Buckner, D., Yu, J., et al. (2012). Ontogeny of the maize shoot apical meristem. Plant Cell 24, 3219-3234.

Tanaka, T., Koyanagi, K. O., and Itoh, T. (2009). Highly diversified molecular evolution of downstream transcription start sites in rice and Arabidopsis. Plant Physiol. 149, 1316-1324.

The Arabidopsis Genome Initiative. (2000). Analysis of the genome sequence of the flowering plant Arabidopsis thaliana. Nature 408 796-815.

Tohge, T., and Fernie, A. R. (2012). Co-expression and coresponses: within and beyond transcription. Front. Plant Sci. 3:248. doi: 10.3389/fpls.2012.00248

Wang, L., Si, Y., Dedow, L. K., Shao Y., Liu, P., and Brutnell, T. P. (2011). A low-cost library construction protocol and data analysis pipeline for Illumina-based strand-specific multiplex RNA-seq. PLoS ONE 6:e26426. doi: 10.1371/journal.pone.0026426

Wang, S., Wang, X., He, Q., Liu, X., Xu, W., Li, L., et al. (2012). Transcriptome analysis of the roots at early and late seedling stages using Illumina pairedend sequencing and development of EST-SSR markers in radish. Plant Cell Rep. 31, 1437-1447.

Wang, Z., Gerstein, M., and Snyder, M. (2009). RNA-Seq: a revolutionary tool for transcriptomics. Nat. Rev Genet. 10, 57-63.

Weber, A. P. M., Weber, K. L., Carr, K., Wilkerson, C., and Ohlrogge, J. B. (2007). Sampling the Arabidopsis transcriptome with massively parallel pyrosequencing. Plant Physiol. 144, $32-42$.

Xia, R., Zhu, H., An, Y.-q., Beers, E. P., and Zongrang, L. (2012). Apple miRNAs and tasiRNAs with novel regulatory networks. Genome Biol. 13, R47.

Yang, X., Zhang, H., and Li, L. (2012). Alternative mRNA processing increases the complexity of microRNA-based gene regulation in Arabidopsis. Plant J. 70, 421-431.

Zamore, P. D., and Haley, B. (2005). Ribo-gnome: the big world of small RNAs. Science 309, 1519-1524.

Zemach, A., Kim, M. Y., Silva, P., Rodrigues, J. A., Dotson, B., Brooks, M. D., etal. (2010). Local DNA hypomethylation activates genes in rice endosperm. Proc. Natl. Acad. Sci. U.S.A. 107, 18729-18734.

Zenoni, S., Ferrarini, A., Giacomelli, E., Xumerle, L., Fasoli, M., Malerba G., et al. (2010). Characterization of transcriptional complexity during berry development in Vitis vinifera using RNA-Seq. Plant Physiol. 152 1787-1795.

Zhang, H., Wei, L., Miao, H., Zhang, T., and Wang, C. (2012). Development and validation of genic-SSR markers in sesame by RNA-seq. BMC Genomics 13:316. doi: 10.1186/14712164-13-316

Zhang, J., Xu, Y., Huan, Q., and Chong, K. (2009). Deep sequencing of Brachypodium small RNAs at the global genome level identifies microRNAs involved in cold stress response. BMC Genomics 10:449. doi: 10.1186/1471-2164-10-449

Zhang, M., Zhao, H., Xie, S., Chen, J., Xu, Y., Wang, K., et al. (2011). Extensive, clustered parental imprinting of protein-coding and noncoding RNAs in developing maize endosperm. Proc. Natl. Acad. Sci. U.S.A. 108, 20042-20047.

Zhelyazkova, P., Sharma, C. M., Förstner, K. U., Liere, K., Vogel, J. and Börner, T. (2012). The primary transcriptome of barley chloroplasts: numerous noncoding RNAs and the dominating role of the plastidencoded RNA polymerase. Plant Cell 24, 123-136.

Zheng, Q., Ryvkin, P., Li, F., Dragomir, I., Valladares, O., Yang, J., et al. (2010). Genome-wide double-stranded RNA sequencing reveals the functional significance of base-paired RNAs in Arabidopsis. PLoS Genet. 6:e1001141. doi: 10.1371/journal.pgen.1001141

Zhong, S., Fei, Z., Chen, Y.-R., Zheng, Y., Huang, M., Vrebalov, J., et al. (2013). Single-base resolution methylomes of tomato fruit development reveal epigenome modifications associated with ripening. Nat. Biotechnol. 31, 154-159.

Zhong, S., Joung, J.-G., Zheng, Y., Chen, Y.-R., Liu, B., Shao, Y., et al. (2011). High-throughput illumina strand-specific RNA sequencing library preparation. Cold Spring Harb. Protoc. 8, 940-949.

Zhu, Q.-H., and Wang, M.-B. (2012). Molecular functions of long noncoding RNAs in plants. Genes 3, 176-190.

Zhu, W., Schlueter, S. D., and Brendel, V. (2003). Refined annotation of the Arabidopsis genome by complete expressed sequence tag mapping. Plant Physiol. 132, 469-484.

Conflict of Interest Statement: The authors declare that the research was conducted in the absence of any commercial or financial relationships that could be construed as a potential conflict of interest.

Received: 05 February 2013; accepted: 10 March 2013; published online: 01 April 2013.

Citation: Martin LBB, Fei Z, Giovannoni JJ and Rose JKC (2013) Catalyzing plant science research with RNA-seq. Front. Plant Sci. 4:66. doi: 10.3389/fpls. 2013.00066

This article was submitted to Frontiers in Plant Systems Biology, a specialty of Frontiers in Plant Science.

Copyright (๑) 2013 Martin, Fei, Giovannoni and Rose. This is an openaccess article distributed under the terms of the Creative Commons Attribution License, which permits use, distribution and reproduction in other forums, provided the original authors and source are credited and subject to any copyright notices concerning any third-party graphics etc. 